\title{
Anatomopatologia de amostras de bovinos alimentados com glicerol
}

\author{
Anatomic-pathological samples of cattle fed with glycerol
}

\author{
Jean Pacheco LeãoI Adriano Tony RamosiI Viviane Mayumi MaruoII \\ Domenica Palomaris Mariano de SouzaII José Neuman Miranda Neiva ${ }^{\mathrm{I}{ }^{*}}$ João Restle ${ }^{\mathrm{II}}$ \\ Sandro Estevan Moron ${ }^{\text {III }}$
}

\begin{abstract}
O aumento na produção de biodiesel vem RESUMO acompanhado do incremento na geração de subprodutos, dentre os quais a glicerina bruta e o glicerol, que vem sendo objeto de inúmeros trabalhos em que se destaca seu uso como fonte energética para alimentação de animais de produção. Entretanto, sua utilização tem sido observada com atenção devido à presença de contaminantes como o metanol, utilizado no processo de separação dos ácidos graxos do glicerol. O metanol é um álcool e tem sido responsável por intoxicações, principalmente em seres humanos e primatas, caracterizadas por acidose metabólica e problemas oculares, apresentando menor toxidade a outras espécies animais. Os efeitos tóxicos da intoxicação por metanol são atribuídos principalmente ao ácido fórmico, um metabólito do processo de degradação do metanol no fígado. Objetivou-se com esse estudo verificar a possível ação tóxica da glicerina bruta e do metanol por análises histopatológicas do sistema nervoso central (SNC), fígado, rúmen, intestino delgado e rim de 24 bovinos confinados por 84 dias, consumindo rações contendo até $24 \%$ de glicerina bruta na matéria seca (MS), a qual apresentava $80,35 \%$ de glicerol, e <0,01\% de metanol. À análise histopatológica, não foram observadas alterações patológicas em amostras provenientes de vacas e novilhos de raças de aptidão leiteira com até $24 \%$ de inclusão de glicerol com base na MS, da dieta. O ganho de peso total e a conversão alimentar não foram afetados pela inclusão da glicerol. Conclui-se que a glicerina bruta pode ser incluída até 24\% da MS total nas dietas de novilhos e vacas de aptidão leiteira sem comprometer o desempenho dos animais e apresentar toxicidade.
\end{abstract}

Palavras-chave: ácido fórmico, acidose metabólica, biodiesel, glicerol

\section{ABSTRACT}

The increase in biodiesel production has led to higher production of crude glycerin, thus, numerous studies have highlighted the use of crude glycerin and glycerol as energy source to be used for animal feed production. However its use has been closely watched because of the presence of contaminants such as methanol used in the process of separating fatty acids from glycerol. Methanol is an alcohol and has been responsible for intoxication in humans and primates, characterized by metabolic acidosis and eye problems, with less toxicity to other species. The toxic effects of methanol poisoning are attributed mainly to formic acid, a metabolite of the degradation process of methanol in the liver. The objective of this study was to verify the possible toxic action of crude glycerin and methanol by pathological examinations of the central nervous system (CNS), liver, rumen, small intestine and kidney from 24 feedlot cattle for 84 days, feed containing up to $24 \%$ of crude glycerin in dry matter (DM) presenting in its composition $80.35 \%$ glycerol, and $<0.01 \%$ methanol. There were no pathological changes observed in dairy cows and steers up to $24 \%$ of glycerol inclusion based on the diet DM. The total weight gain and feed conversion were not affected by inclusion of glycerol. We conclude that crude glycerin can be included up to $24 \%$ of total DM in the diets of steers and dairy cows without compromising animal performance or display toxicity.

Key words: biodiesel, formic acid, glycerol, metabolic acidosis.

A ampliação da produção do biodiesel em substituição parcial ao diesel fóssil tem gerado subprodutos que podem agregar valor e constituir

IEJEPLAN consultoria, Araguaína, TO, Brasil.

IEscola de Medicina Veterinária e Zootecnia, Universidade Federal do Tocantins (UFT), BR-153, Km 112, 77804-970, Zona

Rural, Araguaína, TO, Brasil. E-mail: araguaia2007@gmail.com.*Autor para correspondência.

IIISetor Cimba, (UFT), Araguaína, TO, Brasil. 
alternativas energéticas para alimentação animal, como a glicerina bruta/glicerina (DONKIN, 2008). Por meio do processo de transesterificação metílica, na qual os óleos vegetais ou gordura animal são misturados com metanol associado a um catalizador (ácido ou básico), o biodiesel é produzido e a glicerina bruta é extraída com teores de glicerol podendo variar de 46,48 a 89,2\% (LAGE et al., 2010; ZACARONI, 2010).

Em ruminantes, após a ingestão do glicerol, o mesmo pode ser absorvido pela parede ruminal ou seguir para o intestino onde será absorvido pelas células intestinais seguindo para o fígado onde, pela ação da enzima glicerol quinase, será convertido juntamente com o ATP em glicerol-3-fosfato e ADP, uma etapa intermediaria, onde o glicerol é direcionado para a produção de glicose (LIN, 1977). Outra via de utilização do glicerol se dá por meio de sua fermentação pelas bactérias no rúmen transformando-o principalmente em propionato que será convertido em glicose no fígado (RÉMOND et al., 1993).

Quanto ao poder de intoxicação pela ingestão de glicerol, um caso já foi relatado em humano ingerindo altas quantidades do mesmo, provocando alterações com sinais neurológicos 4 h após a ingestão de 3,88-3,95g de glicerol $\mathrm{kg}^{-1}$ peso corporal, ou seja, mais que o dobro da dose máxima recomendada que é de $1,5 \mathrm{~g} \mathrm{~kg}^{-1}$ de peso corporal (ANDRESEN et al., 2009).

Normalmente, alterações patológicas ligadas ao glicerol são observadas em animais de laboratório submetidos a sua administração por via intramuscular, produzindo rabdomiólise. MARTIM et al., 2007).

Maior atenção tem sido dada à adição de glicerol às dietas de animais de produção, devido à presença de contaminantes como o metanol, utilizado no processo de separação do glicerol dos ácidos graxos. No Brasil, o Ministério da Agricultura, Pecuária e Abastecimento -MAPA, liberou a glicerina bruta para nível máximo de inclusão de $12 \%$ da matéria seca das dietas (MAPA, 2010). A justificativa para tal recomendação é a falta de estudos que comprovem a possibilidade de uso de níveis mais elevados de forma segura.

No presente estudo visou-se avaliar a aplicabilidade da inclusão da glicerol na alimentação de bovinos de raças de aptidão leiteira destinados ao abate. O estudo foi realizado na Escola de Medicina Veterinária e Zootecnia da Universidade Federal do Tocantins, localizada no município de Araguaína-TO. Para um grupo de bovinos de raças de aptidão leiteira, composto por 12 vacas e 12 novilhos castrados com peso vivo médio inicial de 466 e $347 \mathrm{~kg}$ respectivamente. Divididos em oito lotes e mantidos em confinamento por 84 dias, foi fornecido dietas isoprotéica e isoenergética na relação de $20 \%$ de volumoso e $80 \%$ de concentrado com a inclusão de 0, 6, 12 e 24\% com base na matéria seca (MS) de glicerol. O glicerol (GENPA - Glicerol energético Nutricional para Alimentação Animal) utilizado nesse trabalho apresentava na sua composição (\% da matéria seca) $10,08 \%$ de umidade, 1,19\% de extrato etéreo (EE), 7,86\% de matéria mineral (MM), 80,35\% de glicerol, $<0,01 \%$ de metanol e $7,47 \%$ de cloretos, isento de contaminantes, densidade de $1,27 \mathrm{~g} \mathrm{~cm}^{-3}$ e tem registro no Ministério da Agricultura Pecuária e Abastecimento (MAPA) sob número RS-15127 e com limitação de uso para alimentação animal de $12 \%$ da matéria seca total da dieta.

Ao final do período experimental todos os animais foram abatidos em frigorífico comercial da região e coletadas amostras de cérebro, fígado, rim, porção inicial do intestino delgado e rúmen e acondicionadas em formol tamponado, para avaliações histológicas pela coloração com hematoxilina-eosina (HE).

Durante o abate, não foram observadas lesões, condenação ou determinação de aproveitamento condicional das carcaças ou órgãos dos animais. Da mesma forma, não foram encontradas alterações microscópicas nas amostras coletadas. Tais dados indicam que glicerol não apresenta toxicidade para novilhos e vacas nas concentrações testadas.

Os resultados encontrados nesse estudo sugerem que o consumo de glicerol, 24\% na dieta total, não apresenta efeito deletério direto para os bovinos. Entretanto, devido ao fato do mesmo ser fermentado intensamente pelas bactérias do rúmen pode promover o aumento na concentração de ácidos graxos voláteis (DONKIN, 2008), favorecendo assim, a diminuição do pH no rúmen (MACH et al., 2009; WANG et al., 2009) e em dietas mal balanceadas pode ocasionar distúrbios metabólicos e consequentemente provocar injúrias aos ruminantes. PARSONS et al. (2009) testando até $16 \%$ de inclusão de glicerol contendo 85,7\% de glicerol e 0,09\% de metanol na alimentação de 373 novilhas que recebiam dietas com $94 \%$ de concentrado e verificaram que $11,2 \%$ dos animais apresentaram abscessos hepáticos, mas não encontram significância entre o aumento da inclusão do glicerol e a presença dos abscessos. No presente estudo, o glicerol não produziu alterações macroscópicas nem microscópicas e a quantidade de metanol presente no glicerol nos níveis testados foi baixa $(<0,01 \%)$, permitindo possivelmente a completa conversão do mesmo em metano no rúmen (VALADARES FILHO \& PINA, 2006).

A inclusão de glicerol às dietas não afetou o ganho de peso total (GPT) e a conversão alimentar (CA) $(\mathrm{P}>0,05)$, independentemente do sexo dos animais (Tabela 1). Como as dietas fornecidas foram isoprotéicas 
Tabela 1 - Médias, equações de regressão, coeficiente de variação (CV) e nível de significância (P), para as variáveis GPD (kg dia-1 $)$, GPT (kg) e CA em função dos níveis de inclusão de glicerol nas dietas.

\begin{tabular}{|c|c|c|c|c|c|c|c|c|c|}
\hline \multirow{2}{*}{ Parâmetro } & \multirow{2}{*}{ Sexo } & \multicolumn{4}{|c|}{---------Níveis de glicerol ( $\mathrm{g} \mathrm{kg}^{-1} \mathrm{MS}$ )----------- } & \multirow{2}{*}{ Média } & \multirow{2}{*}{ Regressão } & \multirow{2}{*}{ CV(\%) } & \multirow{2}{*}{$P$} \\
\hline & & 0 & 60 & 120 & 240 & & & & \\
\hline \multirow{2}{*}{ GPT (kg) } & VAC & 100,0 & 102,5 & 86,5 & 81,2 & $92,5 \mathrm{a}$ & $\mathrm{Y}=102,2^{\mathrm{NS}}$ & 28,6 & 0,98 \\
\hline & NOV & 104,8 & 104,3 & 109,7 & 88,8 & $101,9 a$ & $\mathrm{Y}=103,5^{\mathrm{NS}}$ & & 0,56 \\
\hline \multirow{2}{*}{ CA } & VAC & $10,1 \mathrm{a}$ & $10,3 a$ & $11,2 \mathrm{a}$ & $12,7 \mathrm{a}$ & 11,1 & $\mathrm{Y}=10,1^{\mathrm{NS}}$ & 26,4 & 0,81 \\
\hline & NOV & $7,7 \mathrm{a}$ & $6,6 a$ & $6,5 b$ & $6,6 b$ & 6,8 & $\mathrm{Y}=7,6^{\mathrm{NS}}$ & & 0,63 \\
\hline
\end{tabular}

Médias na mesma coluna no mesmo parâmetro, seguidas de letras distintas, diferem entre si pelo teste de Tukey a 5\% de probabilidade de erro. $^{\text {NS }}$ - não significativo. VAC: Vaca; NOV: Novilho.

e isoenergéticas e não ter sido observado efeito da inclusão de glicerol no CMS tanto de machos quanto de fêmeas, o GPT também não foi afetado pelas dietas.

A conversão alimentar (CA) não foi afetada (Tabela 1) pela inclusão de até $24 \%$ de glicerol às dietas $(\mathrm{P}>0,05)$. Todavia, ao comparar a CA entre os sexos, as vacas do tratamento com inclusão de 12 e $24 \%$ de glicerol foram 69 e $92 \%$, respectivamente, menos eficientes em converter o alimento ingerido em peso corporal. A pior CA das vacas em relação aos novilhos, nos tratamentos contendo 12 e $24 \%$ de inclusão de glicerol pode estar relacionada ao maior peso de entrada e idade mais avançada desta classe no confinamento, proporcionando alterações na composição do ganho de peso quando estes animais se aproximam da maturidade fisiológica, havendo maior deposição de gordura e aumento do gasto com a manutenção (DI MARCO, 1994).

A adição de glicerol em substituição ao milho na alimentação de bovinos leiteiros é segura até o nível de $24 \%$ da matéria seca total da dieta uma vez que não apresenta toxicidade e pode ser utilizada em dietas para bovinos leiteiros adultos visando minimizar os custos com alimentação uma vez que não altera o ganho de peso e a conversão alimentar dos animais.

\section{CONSELHO DE BIOSSEGURANÇA E ÉTICA}

O Trabalho foi conduzido de acordo com as normas éticas e aprovado pela Comissão de Ética no Uso de Animais da Universidade Federal do Tocantins (UFT) sendo protocolado e

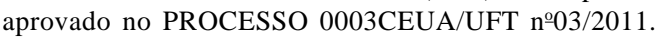

\section{REFERÊNCIAS}

ANDRESEN, H. et al. Severe glycerol intoxication after Menière's disease diagnostic - case report and overview of kinetic data. Clinical Toxicology, v.47, n.4, p.312-316, 2009 Disponível em: <http://informahealthcare.com/doi/abs/10.1080/ 15563650902849123>. Acesso em: 24 ago. 2011. doi. 10.1080/15563650902849123.
DI MARCO, O.N. Crescimiento y respuesta animal. Buenos Aires: Associación Argentina de Producción Animal, 1994. 129p.

DONKIN, S.S. Glycerol from biodiesel production: the new corn for dairy cattle. Revista Brasileira Zootecnia, v.37, supl. esp, p.280-286, 2008. Disponível em: <http:// w w w. s c i e l o.b r / s c i e lo.p h p ? p i d = S 1516 35982008001300032\&script=sci_arttext $>$. Acesso em: 24 ago. 2011. doi.org/10.1590/S1516-35982008001300032.

DONKIN, S.S. et al. Feeding value of glycerol as a replacement for corn grain in rations fed to lactating dairy cows. Journal Dairy Science, n.92, p.5111-5119, 2009. Disponível em: <http://www.journalofdairyscience.org/article/S0022 0302(09)70843-4/abstract>. Acesso em: 24 ago. 2011. doi: 10.3168/jds.2009-2201.

LAGE, J.F. et al. Glicerina bruta na dieta de cordeiros terminados em confinamento. Pesquisa Agropecuária Brasileira, v.45, n.9, p.1012-1020, 2010. Disponível em: <http://www.scielo.br/ scielo.php ? s c ri p t = sci_art text \& pid = S $0100-$ 204X2010000900011\&lng=pt\&nrm=iso>. Acesso em: 24 ago. 2011. doi: org/10.1590/S0100-204X2010000900011.

LIN, E.C.C. Glycerol utilization and its regulation in mammals. Annual Review of Biochemistry, v.46, p765-795, 1977. Disponível em: <http://www.annualreviews.org/doi/abs/10.1146/ annurev.bi.46.070177.004001>. Acesso em: 24 ago. 2011. doi: 10.1146/annurev.bi.46.070177.004001.

MACH, N. et al. Effects of crude glycerin supplementation on performance and meat quality of Holstein bulls fed high-concentrate diets. Journal Animal Science, v.87, p.632-638, 2009. Disponível em: <http://jas.fass.org/cgi/content/full/87/2/632>. Acesso em: 9 nov. 2010. doi: 10.2527/jas.2008-0987.

MARTIM, E.C.O. et al. Lesão renal aguda por glicerol: efeito antioxidante da Vitis vinifera L. Revista Brasileira de Terapia Intensiva, v.19, n.3, p. 292-296, 2007. Disponível em: <http:/ /www.scielo.br/scielo.php?script=sci_arttext\&pid=S0103507X2007000300004_Acesso em: 24 ago. 2011. doi: org/ 10.1590/S0103-507X2007000300004.

MINISTÉRIO DA AGRICULTURA PECUÁRIA E ABASTECIMENTO. GENPA 80 GRANOL. Ingrediente vegetal, Código SIF: RS-15127. Porto Alegre-RS, 2010.

PARSONS, G.L. et al. Performance and carcass traits of finishing heifers fed crude glycerin. Journal Animal Science, v.87, 
p.653-657, 2009. Disponível em: <http://jas.fass.org/cgi/ content/full/87/2/653>. Acesso em: 9 nov. 2010. doi: 10.2527/ jas.2008-1053.

RÉMOND, B. et al. In vitro and in vivo fermentation of glycerol by rumen microbes. Animal Feed Science and Technology, v.41, p.121-132, 1993. Disponível em: <http:// w w w. sciencedirect.com/s cience/article / p i i 0377840193901184>. Acesso em: 24 ago. 2010. doi:10.1016/ 0377-8401(93)90118-4.

VALADARES FILHO, S.; PINA, D.S. Fermentação ruminal. In: BERCHIELLI, T.T. et al. Nutrição de ruminantes. Jaboticabal: FUNEP, 2006. Cap.6, p.151-182.
WANG, C. et al. Effects of glycerol on rumen fermentation, urinary excretion of purine derivatives and feed digestibility in steers. Livestock Science, n.121, p.15-20, 2009. Disponível em: <http:/www.journals.elsevierhealth.com/periodicals/livsci/ article/S1871-1413(08)00153-4/abstract>. Acesso em: 24 ago. 2011. doi: 10.1016/j.livsci.2008.05.010.

ZACARONI, O.F. Respostas de vacas leiteiras à substituição do milho por glicerina bruta. 2010. $43 \mathrm{f}$. Dissertação (Mestrado em Ciências Veterinárias) - Curso de Pós-graduação em Ciências Veterinárias, Universidade Federal de Lavras, MG. 